\title{
The Hadley Circulation and the Weak Temperature Gradient Approximation
}

\author{
L. M. Polvani And A. H. Sobel \\ Department of Applied Physics and Applied Mathematics, and Department of Earth and Environmental Sciences, Columbia University, \\ New York, New York
}

(Manuscript received 21 June 2001, in final form 2 November 2001)

\begin{abstract}
The weak temperature gradient (WTG) approximation is applied to simple shallow-water models of the Hadley circulation. While it is difficult to formally justify the use of the WTG approximation for this problem, the derived WTG solutions are shown to agree well with numerical solutions of the full equations and to converge to the traditional angular momentum conserving (AMC) solutions in the inviscid limit. Heuristic arguments are given to explain this. The WTG method also provides semianalytical solutions in the case of nonvanishing viscosity, in contrast to the AMC solutions, which are strictly inviscid.
\end{abstract}

\section{Introduction}

Balance models, such as the quasigeostrophic, are at the very foundation of much of our understanding of extratropical atmospheric dynamics. The so-called weak temperature gradient (WTG) approximation has been proposed (Sobel and Bretherton 2000; Sobel et al. 2001; Bretherton and Sobel 2002, manuscript submitted to $J$. Atmos. Sci., hereafter BS02) as a balance model for the Tropics. WTG is somewhat distinct from other balance models in that it does not assume the vorticity to be large compared to the divergence, nor does it assume the motion to be adiabatic at leading order. In addition, the fundamental WTG balance relation-that free tropospheric temperature is horizontally uniform at leading order-constrains not only the large-scale fluid dynamics, per se, but also the diabatic processes.

The aim of the above-mentioned studies has been to move toward a coherent theory for large-scale tropical circulations in which the interaction of diabatic processes and large-scale fluid dynamics is clarified and made relatively tractable. As part of this effort, it is necessary to show that the WTG does not contradict aspects of our understanding of tropical atmospheric dynamics which are already well-established. For present purposes, such understanding may be defined by the existence of a theoretical model which, however idealized, is widely accepted as capturing some fundamental aspect of the tropical circulation. Hence, for instance, it has been shown that the WTG approximation to the Gill model (Gill 1980) retains the basic qualitative

Corresponding author address: Prof. Lorenzo M. Polvani, S. W. Mudd Bldg., Room 216, Columbia University, 500 West 120th St., New York, NY 10027.

E-mail: polvani@columbia.edu features of the original, and in the limit of weak thermal damping is quite a good approximation to it (Neelin 1988; Bretherton and Sobel 2002).

In this paper, we examine the WTG approximation in the simplest nonlinear models of the Hadley circulation, namely those that can be expressed as axisymmetric shallow-water models with the heating parameterized as a Newtonian relaxation on the layer thickness. Such models have been studied by Schneider (1987), Held and Phillips (1990), and Hsu and Plumb (2000), and can be considered further idealizations of the twodimensional axisymmetric models that have been applied more extensively to the Hadley circulation (Schneider 1977; Schneider and Lindzen 1977; Held and Hou 1980; Lindzen and Hou 1988; Hack et al. 1989; Plumb and Hou 1992; Satoh 1994; Fang and Tung 1996, 1997, 1999). The study of Fang and Tung (1996) is particularly relevant to the present work, since those authors exploit the fact that temperature is horizontally uniform within the Hadley cell in order to obtain analytic solutions. Nonetheless, there are significant differences between their approach and ours, which we discuss at the end of section 5 .

We first consider the problem on an $f$ plane; while somewhat unrealistic, this is adequate to demonstrate the method and bring out its basic properties. We set up the problem in the next section, find the traditional "angular momentum conserving" (AMC) solution in section 3, and the WTG solution in section 4. Although the WTG solution is shown to converge to the AMC solution exactly in the inviscid limit, the WTG approximation does not appear to be formally justifiable for this problem. Hence, in the following section, a heuristic discussion lists some of the reasons for the apparent success of 
WTG. We present the equatorial $\beta$ plane case in section 6 , and summarize our conclusions in section 7 .

\section{Statement of the problem}

We consider a rotating fluid on an $f$ plane, obeying the shallow water equations:

$$
\begin{aligned}
u_{t}+v u_{y}-v & =-\alpha u, \\
v_{t}+v v_{y}+u & =-h_{y}-\alpha v, \quad \text { and } \\
h_{t}+(h v)_{y} & =Q,
\end{aligned}
$$

where the $y$ axis is aligned with the meridional direction, $u$ and $v$ are the zonal and meridional velocities, and $h$ $\equiv(1+\eta)$ is the fluid thickness (here referred to as the "temperature"). All fields are assumed to be independent of the zonal coordinate $x$. The system is here nondimensionalized with the Rossby deformation radius as the length scale and $f^{-1}$ as the timescale; $\alpha$ is the nondimensional Rayleigh friction coefficient. Since the shallow water model is best viewed as representing the upper branch of the Hadley circulation (Held and Phillips 1990), the parameter $\alpha$ represents internal, as opposed to surface, friction. While this is likely too small in the real atmosphere, retaining it breaks the constraint of angular momentum conservation and serves to highlight interesting aspects of the WTG approach, though we will also consider the inviscid case $\alpha=0$.

The "thermal" forcing $Q$ is parameterized, in the customary manner, with

$$
Q \equiv \frac{\eta_{E}-\eta}{\tau}
$$

where $\eta_{E}$ is a specified "equilibrium temperature" profile, and $\tau$ some thermal relaxation timescale. For simplicity, we here take $\eta_{E}$ to be a top-hat function that is,

$$
\eta_{E}(y)= \begin{cases}H_{E} & \text { for }|y|<Y_{E} \\ 0 & \text { for }|y|>Y_{E}\end{cases}
$$

Hence $H_{E}$ is a measure of the strength of the forcing, and $Y_{E}$ a measure of the size of the forcing region. As in other similar studies using the shallow water system cited above, we neglect the vertical advection of momentum associated with the mass source $Q$. Also, note that because our choice of $\eta_{E}$ has infinite slope at $y=$ $Y_{E}$, it will always be supercritical in the sense of Plumb and Hou (1992); that is, a circulation will exist for arbitrarily small $H_{E}$. Our aim is to construct time-independent solutions to (1)-(3), representing the steadystate atmospheric response to a localized forcing, but we avoid addressing the threshold behavior that would be associated with a more general, smooth choice of $\eta_{E}$.

\section{The angular momentum conserving solution}

For clarity, we start by deriving the traditional angular momentum conserving solution (denoted by the sub- script $a$ ). Hsu and Plumb (2000) present a solution of this kind for a shallow water system on an $f$ plane with an axisymmetric forcing. While the problem here is rectilinear, the solution method is essentially identical to that of Hsu and Plumb.

Taking $\alpha=0$, one assumes the existence of a latitude $Y_{H}>Y_{E}$-defining the "Hadley cell" width-outside which the flow is nondivergent, $v_{a}=0$, and in thermal equilibrium, $\eta_{a}=\eta_{E}$. In general, this implies that $u_{a}$ is in geostrophic balance with $\eta_{a}$. For the simple choice of $\eta_{E}$ in (5), this implies $u_{a}=0$, so that all fields vanish outside the Hadley cell.

Inside the cell, the flow is assumed to be inviscid ( $\alpha$ $=0$ ) and divergent; that is, $v \neq 0$. For a stationary solution, it then follows from (1) that

$$
u_{a}(y)=y \text {, }
$$

for $|y|<Y_{H}$. Notice that the zonal velocity of the AMC solution is discontinuous at $y=Y_{H}$. Next, the temperature is obtained by dropping the term $v v_{y}$ in (2), so the zonal flow is assumed to be geostrophic. This assumption, a common one in models of the sort discussed here, is not made in the full numerical solutions to which we compare the WTG solutions in section 5. Integrating $u=-\eta_{y}$ one obtains

$$
\eta_{a}(y)=\frac{1}{2}\left(Y_{H}^{2}-y^{2}\right),
$$

where the integration constant is chosen so that $\eta_{a}$ is continuous at $y=Y_{H}$. The meridional velocity is then integrated using (3):

$$
v_{a}(y)=\frac{1}{\left(1+\eta_{a}\right)} \int_{0}^{y} \frac{\eta_{E}\left(y^{\prime}\right)-\eta_{a}\left(y^{\prime}\right)}{\tau} d y^{\prime} .
$$

Finally, the width $Y_{H}$ of the cell is obtained by enforcing zero net mass across $y=Y_{H}$, which is equivalent to setting

$$
\int_{0}^{Y_{H}} \frac{\eta_{E}(y)-\eta_{a}(y)}{\tau} d y=0 .
$$

Substituting $\eta_{E}$ from (5) and $\eta_{a}$ from (7), one easily obtains

$$
Y_{H}=\left(3 H_{E} Y_{E}\right)^{1 / 3} \text {. }
$$

The $u, v$, and $\eta$ fields of the AMC solution are illustrated by the heavy solid lines in Fig. 2, later.

\section{The weak temperature gradient (WTG) solution}

The crux of the WTG approximation is that the divergence $\delta$ of the flow is directly obtained from the heating $Q$ since temperature gradients may be neglected in solving (3). Letting

$$
\eta(y)=\eta_{0}+\eta_{1}(y)
$$

inside the cell, and neglecting all terms involving $\eta_{1}$, the continuity Eq. (3) is approximated under WTG by 


$$
\left(1+\eta_{0}\right) \delta=\frac{\eta_{E}-\eta_{0}}{\tau} .
$$

The neglect of $\eta_{1}$ in (12) is not formally justifiable using the scaling arguments presented by Sobel et al. (2001). Those require a flow whose length scale is small compared to the Rossby radius, and our solution for the Hadley cell width will be of the order of the Rossby radius (as it should be). In addition, as we will show, $\eta_{1}$ is not very small compared to $\eta_{0}$ (which is retained) for all $y<Y_{H}$. Hence the validity of (12) is not so easily established. We ask the reader simply to accept it for the sake of argument at this point. Some degree of justification will be provided a posteriori by the solutions obtained, and a heuristic argument will be given in the following section.

The constant $\eta_{0}$ is chosen so that the flow has zero net divergence over the cell, which is required to obtain a vanishing velocity at the edge of the cell. Hence given (12), we require that

$$
\int_{0}^{Y_{H}}\left(\frac{\eta_{E}-\eta_{0}}{\tau}\right) d y=0
$$

which yields

$$
\eta_{0}=H_{E} \frac{Y_{E}}{Y_{H}}
$$

The Hadley cell width $Y_{H}$ is as yet unspecified. It should be clear that, as in the AMC case, all fields vanish for $|y|>Y_{H}$.

Solving (12), the divergence takes the simple form,

$$
\delta(y)= \begin{cases}q_{0} & \text { for }|y|<Y_{E} \\ q_{\kappa} & \text { for } Y_{E}<|y|<Y_{H},\end{cases}
$$

where $q_{0}=\left(H_{E}-\eta_{0}\right) /\left[\tau\left(1+\eta_{0}\right)\right], q_{\kappa}=\kappa q_{0}$, and $\kappa$ $=\left(1-Y_{H} / Y_{E}\right)^{-1}$ is a small negative number chosen so that the integral of $\delta$ over the cell vanishes. Integrating this divergence yields the meridional velocity,

$$
v(y)= \begin{cases}q_{0} y & \text { for }|y|<Y_{E} \\ q_{\kappa}\left(y-Y_{H}\right) & \text { for } Y_{E}<|y|<Y_{H} .\end{cases}
$$

Note that $v$ vanishes at the edge of the cell $\left(y=Y_{H}\right)$, and is continuous at the edge of the heating region $y=$ $Y_{E}$.

The second step in the WTG solution consists of finding a time-independent solution to the vorticity equation

$$
\zeta_{t}+[v(\zeta+1)]_{y}=-\alpha \zeta .
$$

With the divergent velocity $v$ from (16), the vorticity $\zeta$ is easily obtained:

$\zeta(y)= \begin{cases}\zeta_{0} & \text { for }|y|<Y_{E} \\ \zeta_{\kappa}+\left(\zeta_{0}-\zeta_{\kappa}\right)\left(\frac{y-Y_{H}}{Y_{E}-Y_{H}}\right)^{\left(1 / \zeta_{\kappa}\right)} & \text { for } Y_{E}<|y|<Y_{H}\end{cases}$

where $\zeta_{0}=-\left(1+\alpha / q_{0}\right)^{-1}$, and $\zeta_{\kappa}=-\left(1+\alpha / q_{\kappa}\right)^{-1}$. In the WTG approximation, we construct the vorticity $\zeta$ so that it is continuous at $y=Y_{E}$. This yields $\zeta=\zeta_{\text {k }}$ at $y=Y_{H}$, implying a kink in the associated rotational zonal velocity $u$ at the edge of the Hadley cell (recall that in AMC solution $u$ is actually discontinuous there). The zonal velocity $u$ is obtained by integrating $\zeta=-u_{y}$, which yields,

$$
u(y)= \begin{cases}-\zeta_{0} y & \text { for }|y|<Y_{E} \\ \zeta_{\kappa}\left(Y_{H}-y\right)+\left(\zeta_{0}-\zeta_{\kappa}\right)\left(\frac{q_{0} Y_{E}}{\alpha}\right)\left(\frac{y-Y_{H}}{Y_{E}-Y_{H}}\right)^{-\left(\alpha / q_{k}\right)} & \text { for } Y_{E}<|y|<Y_{H} .\end{cases}
$$

It is worth noting that both $u$ and its first derivative are continuous at the edge of the forcing region, unlike $v$, which is not differentiable at $y=Y_{E}$.

Having obtained both $u$ and $v$, we can now compute spatial variations of temperature- that is, $\eta_{1}(y)$-by integrating (2). Neglecting the small term $v v_{y}$, as in the AMC solution, this yields

$$
\eta_{1}(y)=-\eta_{0}+\int_{y}^{Y_{H}}\left[u\left(y^{\prime}\right)+\alpha v\left(y^{\prime}\right)\right] d y^{\prime},
$$

where the integration constant has been chosen to ensure continuity of temperature at the edge of the Hadley cell; that is, $\eta\left(Y_{H}\right)=\eta_{0}+\eta_{1}\left(Y_{H}\right)=0$.

Finally, as in the AMC solution, the width of the
Hadley cell is obtained by requiring that the net forcing over the cell vanish; that is,

$$
\int_{0}^{Y_{H}} Q(y) d y=0
$$

From the definition (4), and the fact that $\eta_{0}$ was chosen to satisfy (13), it follows that we must require that

$$
\int_{0}^{Y_{H}} \eta_{1}(y) d y=0
$$

This equation can be thought of as our implicit definition of $Y_{H}$. Unfortunately, the high nonlinearity of the expressions-notice that $Y_{H}$ appears even in the exponent 


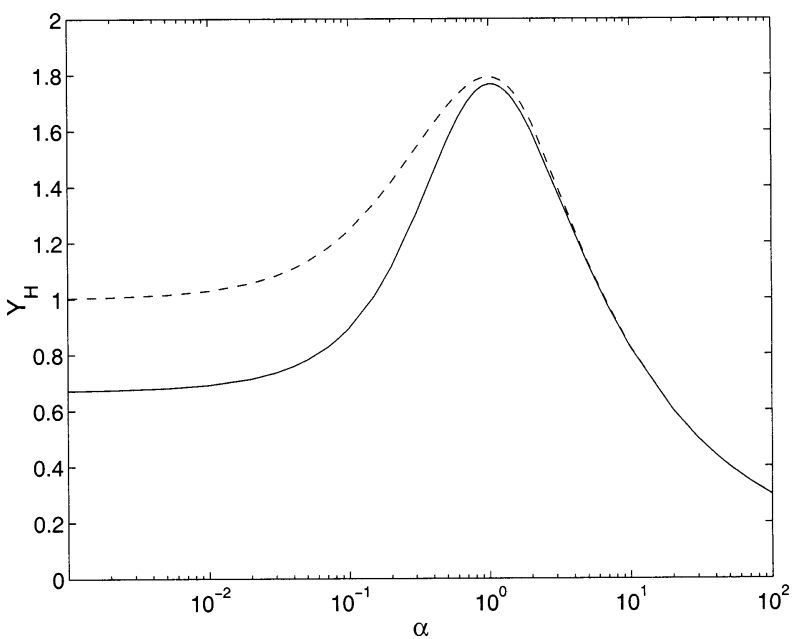

FIG. 1. The Hadley cell width $Y_{H}$ (solid line) as a function of the dissipation $\alpha$ for the case $H_{E}=1, Y_{E}=0.1, \tau=1$; in the limit $\alpha$ $\rightarrow 0$, the curve asymptotes to the AMC value $\left(3 H_{E} Y_{E}\right)^{(1 / 3)} \approx 0.6694$. The dashed line shows the width $Y_{H}$ for the case with an equatorial $\beta$ plane.

in (19)—-makes it rather difficult to extract from (22) even an approximate formula for $Y_{H}$. It is, however, a simple matter to compute $Y_{H}$ numerically.

As an illustration, we show in Fig. 1 the values of $Y_{H}$ as a function of the dissipation $\alpha$, for a representative set of values $H_{E}=1, Y_{E}=0.1, \tau=1$. Note how the Hadley cell width reaches a maximum for $\alpha \approx O(1)$. We interpret this as follows. The extent of the Hadley cell is ultimately set by the requirement that $Q$ integrate to zero over the cell. Simultaneously, continuity of $\eta$ requires $\eta_{1}=-\eta_{0}$ at $Y_{H}$, and for given $Y_{H}, \eta_{0}$ is fixed by (14), hence $\eta_{1}\left(Y_{H}\right)$ is also fixed. In this situation, anything that reduces the magnitude of the (negative) slope $\eta_{y}$ will increase the width of the cell. Dissipation clearly decreases $u$ relative to the inviscid case, thus reducing the (nearly) geostrophically balanced slope. Hence, a small amount of dissipation increases the cell width compared to the inviscid case. This behavior is also seen in the numerical solutions on the sphere presented by Held and Phillips (1990).

On the other hand, when too much dissipation is present, the circulation generated by the forcing becomes very weak, requiring the Hadley cell width to shrink for $\alpha \gg 1$. Hence the occurrence of a maximum, which happens around $\alpha \approx 1.033 \ldots$, for this choice of parameters. A maximum cell size near $\alpha=1$ is also evident in the full numerical solutions, presented in the next section, though we do not show solutions for $\alpha>$ 1 . In any case, large values of $\alpha$ are not particularly relevant, since our simple shallow water model should really be thought of as a representation of the upper tropospheric branch of the circulation, which is presumably at most weakly viscous. Further, the WTG approximation is not expected to be valid for large friction
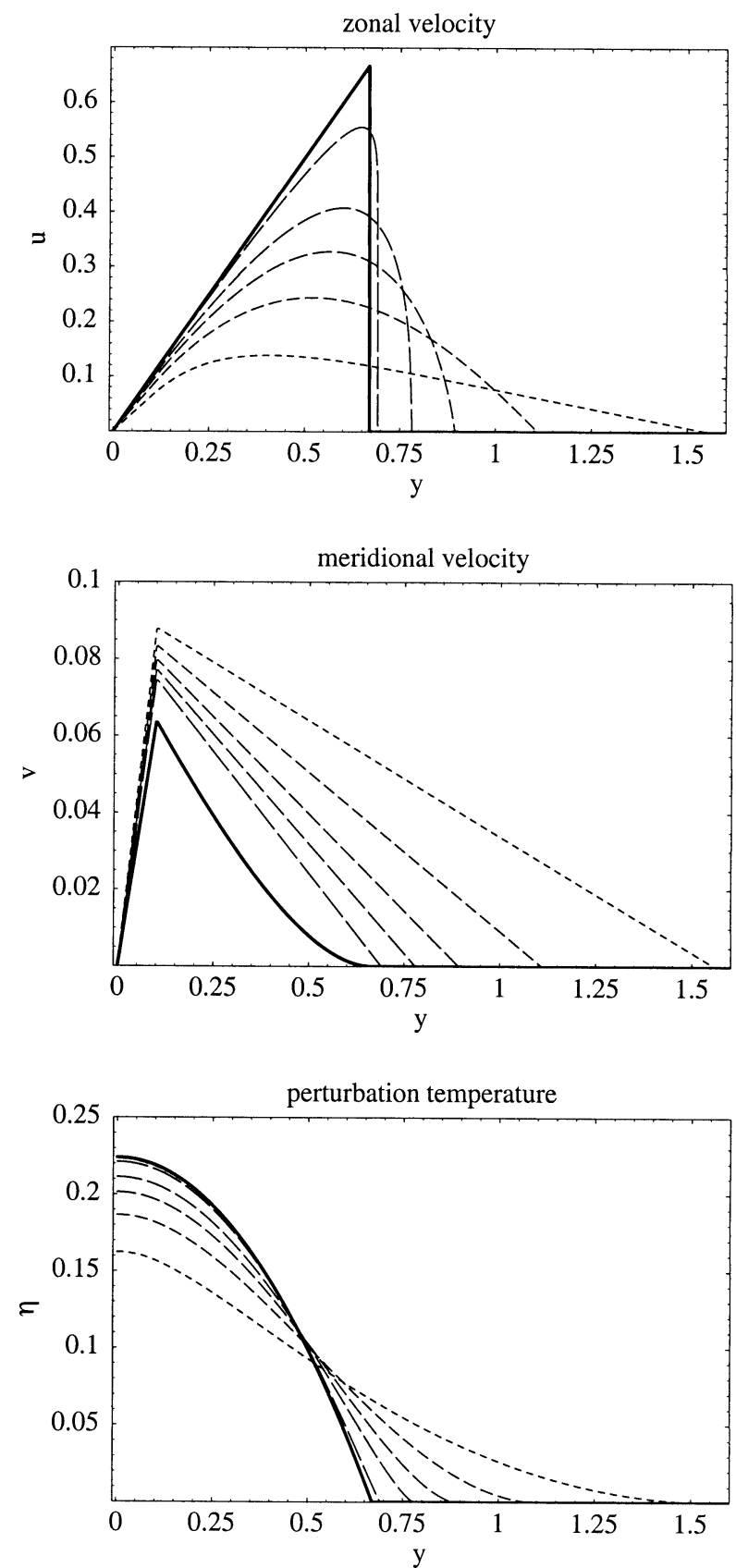

FIG. 2. The WTG solutions for $\alpha=0.01,0.05,0.1,0.2$, and 0.5 . Longer dashes correspond to smaller values of $\alpha$. The thick solid line is the AMC solution. For all curves, $H_{E}=1, Y_{E}=0.1, \tau=1$.

because the effect of friction on the divergent flow is entirely neglected under WTG (Sobel et al. 2001).

As can be seen in Fig. 1, in the limit $\alpha \rightarrow 0$ the Hadley cell width obtained in the WTG approximation asymptotes to a constant value: this is precisely the inviscid AMC value given by (10). In fact, both the zonal velocity $u$ and the perturbation temperature $\eta$ converge to the AMC solution in the limit $\alpha \rightarrow 0$, as shown in Fig. 2. This is not surprising since, in that limit, the 

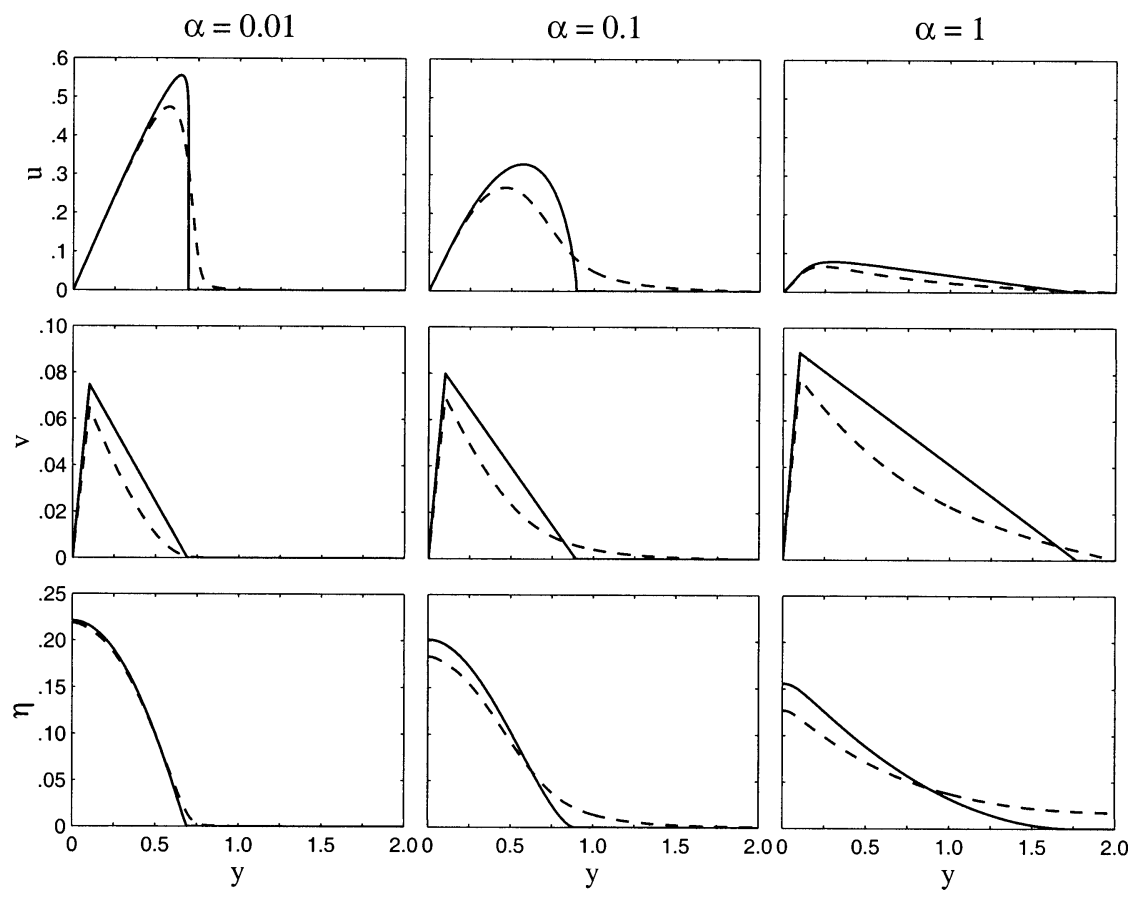

FIG. 3. The WTG solutions (solid curves) and the full solutions (dashed curves) for $\alpha=0.01$, 0.1 , and 1. For all curves, $H_{E}=1, Y_{E}=0.1, \tau=1$.

WTG solution (18) for the vorticity tends ${ }^{1}$ to $\zeta(y)=$ -1 , the zero absolute vorticity AMC solution. Hence $u$ $\rightarrow u_{a}$ and, since $\eta$ comes from the linear geostrophic balance (2), $\eta \rightarrow \eta_{a}$ as $\alpha \rightarrow 0$.

The meridional velocity $v$, in contrast, does not converge to the AMC limit as $\alpha \rightarrow 0$. This is because in the WTG solution, $v$ is computed from the divergence $\delta$, which is obtained directly from the forcing $Q$ using the constant value $\eta_{0}$ [cf. Eq. (12)]. This latter value can be thought of as the "mean" perturbation temperature, and is clearly smaller than the actual perturbation temperature in the forcing region $|y|<Y_{E}$. This underestimate of $\eta$ as it enters $Q$ results in the WTG solution having a stronger forcing than it should, and yields larger values of $v$ even for $\alpha=0$. However, the WTG solution can be used to compute the exact AMC divergent flow as a higher-order correction. Since the total WTG $\eta=\eta_{0}+\eta_{1}$ is identical to the AMC $\eta$ in the inviscid limit, if that $\eta$ is used in (8), the AMC solution for $v_{a}$ will also be reproduced exactly.

Finally, we remark that the approach to the AMC solution as $\alpha \rightarrow 0$ is somewhat richer that might be naively expected. Close inspection of (18) reveals that the WTG solution for the vorticity $\zeta$ becomes singular at $y=Y_{H}$ when $\alpha$ is smaller than the critical value $\alpha_{c}$ $=-q_{\kappa}$ (at which value $\zeta_{\kappa}=0$ ). Expression (18) is in fact valid for all $\alpha \neq \alpha_{c}$; at the critical value $\zeta$ has a logarithmic term. What happens as the critical dissipation value $\alpha_{c}$ is crossed can be seen in the top panel

\footnotetext{
${ }^{1}$ With a very steep boundary layer at the edge of the cell, $y=Y_{H}$.
}

of Fig. 2: the vorticity, which is the slope of $u$, becomes infinite at the edge of the cell for $\alpha \leq \alpha_{c}$. For the parameters of Fig. 2, we find $\alpha_{c} \approx 0.1006 \ldots$ Mathematically, a singularity as $\alpha \rightarrow 0$ is expected, since the WTG solution needs to approach the discontinuous AMC solution in that limit. However, we do not at present have an intuitive understanding for the occurrence of the singularity at a finite value of $\alpha$.

\section{Discussion}

\section{a. Comparison with the full solutions}

To demonstrate that the WTG solution is a good approximation to the full solution of the Hadley cell problem, we have solved (1)-(3) numerically using a shooting method. These full solutions are presented in Fig. 3 , together with the WTG solutions, for three representative values of the dissipation $\alpha(0.01,0.1$, and 1.0). Surprisingly, even for $\alpha=1$ the WTG solution agrees well, both quantitatively and qualitatively, with the numerical solution. The most significant difference is perhaps the lack of a precisely defined cell size in the numerical solutions, as all fields approach zero in a smooth manner.

\section{b. Reasons for the validity of the WTG approximation}

As noted in the derivation of the WTG solution, the key approximation, the neglect of $\eta_{1}$ in the computation of the divergence, does not appear formally justifiable, 
yet we see good agreement of the WTG and numerical solutions. This requires some discussion.

We first note that there is an important difference between the present nonlinear Hadley circulation problem and linear WTG problems solved in earlier studies (Neelin 1988; Sobel et al. 2001; BS02). In those problems, while the thickness perturbation (here $\eta$ ) could be computed as a small correction to the constant mean $h$, doing so was unnecessary in order to compute the flow. We might think of those problems as yielding to a "strict" WTG approximation in which the temperature is of entirely secondary importance. Here, while $\eta$ is still computed as a correction to an $h$ field that is (piecewise) constant to leading order, the problem cannot in fact be closed without computing that correction.

It is fairly straightforward to explain why, in the inviscid limit, the WTG solution must converge to the AMC solution. In both solutions, as $\alpha \rightarrow 0, u$ and $\eta$, and hence also $Y_{H}$, can be thought of as being directly implied by a state of zero absolute vorticity in the Hadley cell, which, in an axisymmetric framework, corresponds to constant angular momentum. In that limit, the details of the divergence-which is where the error first appears in the WTG solution-become unimportant, since the vortex-stretching effect vanishes. Once a zero absolute vorticity solution has been assumed, the rest of the WTG solution procedure is in fact formally identical to the AMC procedure.

The case of small but nonzero $\alpha$ can be addressed by a generalization of the above argument. The neglect of $\eta_{1}$ (until the end of the WTG calculation) has an impact through the resultant error in the heating and divergence. Near $y=0$, this is not particularly problematic. Since our length scale is (locally) roughly equal to $|y|$, which is much smaller than the Rossby radius, and since the heating is large here, WTG is formally valid. It is still inconsistent to keep $\eta_{0}$ while neglecting $\eta_{1}$, but since both are small compared to $\eta_{E}$, the error is nonetheless similarly small. For $|y|>Y_{E}$, we appear at first to have a more serious problem. Because the heating is small in this region, and $|y|$ approaches the Rossby radius, WTG is not formally valid. Furthermore, in this region the heating is of order $\eta$. Since $\eta_{0}$ and $\eta_{1}$ are of the same order, keeping one while neglecting the other results in an error in the heating that is of the order as the local heating itself. The reason this does not have catastrophic consequences is that in this region the heating is small and thus, for small viscosity, the vorticity dynamics is nearly conservative and the solution is controlled predominantly by vorticity advection (which drives this region to zero absolute vorticity in the inviscid case). The WTG vorticity equation reduces to the barotropic nondivergent vorticity equation in the limit of vanishing heating, and while this distorts the true adiabatic dynamics somewhat (because the primitive shallow water system does allow divergence even for zero mass source), even at latitudes as high as the subtropics this error is not extremely large because height gradients are still modest. Hence not only the inviscid but also the nearly inviscid solution turn out to be fairly accurate under WTG.

\section{c. Comparison with Satoh (1994) and Fang and Tung (1996)}

As mentioned in the introduction, Satoh (1994) and Fang and Tung (1996) computed solutions to a twodimensional (latitude-height) axisymmetric Hadley circulation problem in which they explicitly used the constraint of horizontally uniform temperature within the Hadley cell, allowing them to diagnose the heating and divergent flow within the cell without any explicit dependence on the rest of the solution (see their section $4 c$ ). Because of this fundamental similarity, it is instructive to compare this procedure with ours in order to see how these complementary studies illuminate different aspects of the Hadley problem.

The most obvious difference between these two earlier studies and ours is of course our reduced dimensionality due to use of the shallow water system, and the consequent greater sophistication and realism of their solutions. Our shallow water formulation and very idealized choice for $\eta_{E}$ render the present study much cruder than Satoh's or Fang and Tung's as a representation of the Hadley circulation. However, in other respects our approach is more general and places the Hadley circulation in the context of a broader theory for tropical atmospheric dynamics.

One difference, perhaps minor, between Fang and Tung's study and ours is that their simulation assumed the existence of an infinitely narrow (in latitude) intertropical convergence zone, in which the atmospheric temperature was locked to that of the surface by a hard convective adjustment or "strict quasi-equilibrium" (Emanuel et al. 1994) assumption. This is equivalent to taking the relaxation timescale $\tau=0$ [in Eq. (4)] at a single latitude, but a constant finite value elsewhere. This fixes the temperature profile at one latitude, and by horizontal uniformity immediately determines the temperature throughout the Hadley cell, independent of the cell width or any other aspect of the rest of the solution. In the present study, by contrast, $\tau$ is finite everywhere and even the leading order temperature $\eta_{0}$ must be determined as part of the full solution. This approach generalizes to arbitrary convective closures in which the adjustment will not be exactly instantaneous and radiative cooling, in particular, will be able to modify the temperature. In practice this is probably a small effect since we generally expect the convective timescale, however defined, to be short relative to the radiative timescale.

A more significant difference lies in the determination of the zonal wind. Satoh (1994) and Fang and Tung (1996) used the principle of homogenization of angular momentum in the Hadley cell, which is valid only for inviscid, steady, axisymmetric flow. We instead solve a 
vorticity equation in order to obtain the zonal wind, which requires none of these assumptions.

Related to this, Satoh and Fang and Tung obtained their constraints of horizontally uniform temperature in the Hadley cell as a consequence of the homogenization of angular momentum. A uniform value of angular momentum means that the zonal flow is a function only of latitude, not height, in the Hadley cell, and thus, by a thermal wind relationship, there is no horizontal temperature gradient. The geopotential gradient required to sustain the zonal wind is not at any stage neglected in Satoh's or Fang and Tung's solution. This gradient exists, despite latitudinal temperature homogenization in the Hadley cell, because of the slope of the cell boundary with height (something which cannot be captured by our shallow water model). Air parcels in the Hadley cell, where temperature is horizontally uniform, overlie parcels at the same latitude which are outside the cell. Since the region outside the cell has a latitudinal temperature gradient, there can be a geopotential gradient within the cell. In contrast, in our approach the horizontal temperature gradient is neglected as an a priori approximation, based on the fact that Coriolis effects are weak in the Tropics. This implies that balanced horizontal geopotential gradients are weak, and assuming small surface pressure gradients, horizontal temperature gradients must also be weak, as is observed to be the case. This WTG approach is approximate rather than exact, but generalizes to viscous, unsteady, and nonaxisymmetric flow. Thus, while the temperature homogenization in Satoh (1994) and Fang and Tung (1996) is a special result applicable to certain idealizations of the Hadley circulation, ours is just an instance of a much more general approximate constraint on a wide range of large-scale tropical flows.

\section{The $\beta$-plane case}

The WTG solution discussed above can be extended from an $f$ plane to an equatorial $\beta$ plane with only minor modifications. In the presence of $\beta$, the momentum Eqs. (1) and (2) become

$$
\begin{aligned}
& u_{t}+v u_{y}-y v=-\alpha u, \quad \text { and } \\
& v_{t}+v v_{y}+y u=-h_{y}-\alpha v,
\end{aligned}
$$

(our nondimensionalization implies $\beta=1$ ). Note that the continuity Eq. (3) is unaffected by $\beta$ and thus, in the WTG approximation, the divergent component of the solution is still given by (15) and (16).

The effect of $\beta$ is felt in the vorticity equation, which becomes

$$
\zeta_{t}+[v(\zeta+y)]_{y}=-\alpha \zeta .
$$

Again this is easily solved and yields

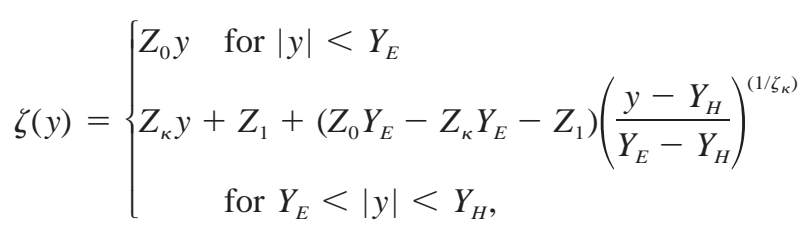

where $Z_{0}=-\left[1+\alpha /\left(2 q_{0}\right)\right]^{-1}, Z_{\kappa}=-\left[1+\alpha /\left(2 q_{\kappa}\right)\right]^{-1}$, and $Z_{1}=q_{\kappa} Y_{H}\left(1+Z_{\kappa}\right)\left(q_{\kappa}+\alpha\right)^{-1}$. As for the $f$-plane case, $\zeta$ is continuous at $y=Y_{E}$, but has a discontinuity at $y=Y_{H}$, implying a small kink in the zonal velocity $u$ at the edge of the Hadley cell.

Integration of (26) yields the rotational velocity $u$ :

$$
u(y)= \begin{cases}-\left(\frac{1}{2}\right) Z_{0} y^{2} & \text { for }|y|<Y_{E} \\ \left(\frac{1}{2}\right) Z_{\kappa}\left(Y_{H}^{2}-y^{2}\right)+Z_{1}\left(Y_{H}-y\right)+\left(Z_{0} Y_{E}-Z_{\kappa} Y_{E}-Z_{1}\right)\left(\frac{q_{0} Y_{E}}{\alpha}\right)\left(\frac{y-Y_{H}}{Y_{E}-Y_{H}}\right)^{-\left(\alpha / q_{\kappa}\right)} & \text { for } Y_{E}<|y|<Y_{H} .\end{cases}
$$

Note that, both $u$ and its first derivative are continuous at the edge of the forcing region.

As for the $f$-plane case, the spatial variations of the temperature field are obtained from $v$-momentum equation, here (24), which yields

$$
\eta_{1}(y)=-\eta_{0}+\int_{y}^{Y_{H}}\left[y^{\prime} u\left(y^{\prime}\right)+\alpha v\left(y^{\prime}\right)\right] d y^{\prime} .
$$

Finally, the radius of the Hadley cell is determined from the constraint (21), which we solve numerically as in the $f$-plane case.
The dashed line in Fig. 1 shows that, in general, the Hadley cell width is greater on the equatorial $\beta$ plane than on the $f$ plane. This is not surprising since it is the Coriolis force that limits the cell width, and the Coriolis parameter is weaker on the equatorial $\beta$ plane than on the $f$ plane.

In Fig. 4 , the $\beta$-plane solutions are plotted, for the representative case $\alpha=0.1$; the $f$-plane solutions are superimposed in dashed lines, for easy comparison. Note that the zonal velocity is quadratic at the origin, as per Eq. (27). This implies, via (28), that $\eta$ is quartic in the forcing region, as is clearly reflected in the very 

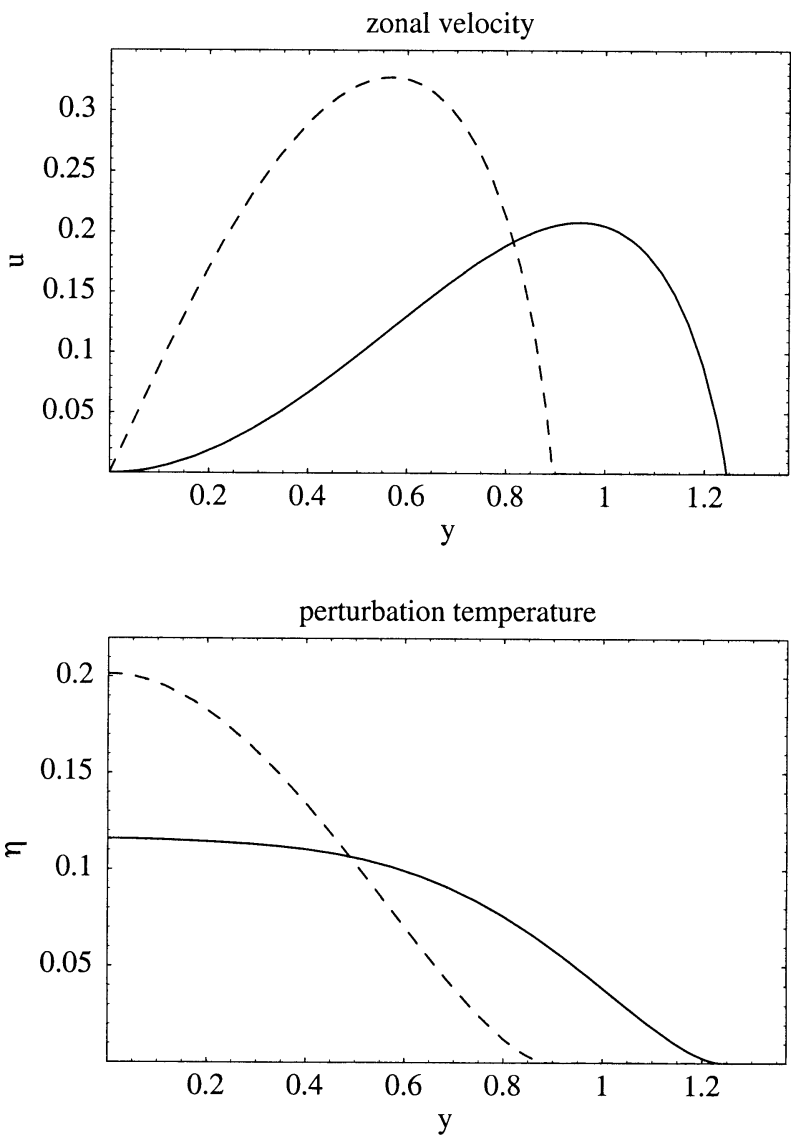

FIG. 4. The WTG solution on an equatorial $\beta$ plane (solid lines) for the case $\alpha=0.1$. The dashed lines show the corresponding $f$ plane solutions. The meridional velocity $v$ is identical (and thus not plotted). For all curves, $H_{E}=1, Y_{E}=0.1, \tau=1$.

flat $\eta$ curve. Hence, the WTG approximation (i.e., the fact that temperature is constant at lowest order) seems even better satisfied on the equatorial $\beta$ plane than on the $f$ plane.

In the inviscid limit, the WTG solution again converges to the AMC solution in the same sense as on the $f$ plane. It is straightforward to show that in the limit $\alpha \rightarrow 0$ the Hadley cell width is given by

$$
Y_{H}=\left(10 H_{E} Y_{E}\right)^{1 / 5} \text {, }
$$

which can be compared to (10), showing that on the equatorial $\beta$ plane the cell is not only wider, but also less sensitive to the forcing parameter than on the $f$ plane. For our choice of parameters, this yields $Y_{H}=$ 1 in the inviscid limit, as seen in Fig. 1.

\section{Conclusions}

We have used a method based on the WTG approximation to obtain nonlinear, axisymmetric solutions to the shallow water equations under a thermal relaxation and Rayleigh friction, representing perhaps the simplest model of the Hadley circulation. We have obtained so- lutions on both the $f$ plane and the equatorial $\beta$ plane. In the inviscid limit, the solution for the rotational flow $u$, perturbation thickness $\eta$, and cell width $Y_{H}$ are identical to those of the standard AMC solution. The divergent flow differs between the two cases, but the AMC divergent flow can be obtained as a next-order correction to the WTG solution. The WTG procedure has the advantage that it offers a conceptually straightforward, semianalytical recipe for directly obtaining a steady solution under finite (but small) viscosity, while the AMC method is applicable only in the strictly inviscid case. The WTG procedure thus allows us, semianalytically, to take the inviscid limit of the solution itself (as opposed the equations, prior to solving them), something that could otherwise be done to our knowledge only by direct numerical integration of the primitive shallow water system.

This perhaps represents only a minor advance in our understanding of the Hadley circulation, per se. As found by $\mathrm{BS} 02$ in the context of the Gill problem, it seems, also, that no dramatic procedural simplification results from the use of WTG. However, we have been successful in showing that no harm is done either, since in the inviscid limit, the WTG solutions reduce to the "exact" solutions of these classic problems. This may be of some conceptual value.

However, it is when (in contrast to the present work) moisture is explicitly modeled, and allowed to affect the heating, that use of the WTG approximation appears to be most advantageous, as shown to some degree by Sobel and Bretherton (2000) and Sobel et al. (2001). In those problems, great simplification arises because WTG explicitly removes diabatic modes which involve heating variations arising from tropospheric temperature variations. For consistency, however, if WTG is used in the moist, thermodynamical aspects of the problem, it should also be used in the "dry" part of the dynamics. Hence it is necessary to show, as we have done here in the context of a simple model of the Hadley circulation, that the dry dynamics is not seriously compromised by WTG.

Acknowledgments. We thank Chris Bretherton and Alan Plumb for discussions; Ed Schneider, K. K. Tung, and two anonymous reviewers for comments that led to improvements; and the NSF and the David and Lucile Packard Foundation for their support. L. M. Polvani wishes to acknowledge the hospitality of the New Jersey Transit Corporation, on whose trains some of this work was performed.

\section{REFERENCES}

Emanuel, K. A., J. D. Neelin, and C. S. Bretherton, 1994: On largescale circulations in convecting atmospheres. Quart. J. Roy. Meteor. Soc., 120, 1111-1143.

Fang, M., and K. K. Tung, 1996: A simple model of nonlinear Hadley circulation with an ITCZ: Analytic and numerical solutions. $J$. Atmos. Sci., 53, 1241-1261. 
, and - 1997: The dependence of the Hadley circulation on the thermal relaxation time. J. Atmos. Sci., 54, 1379-1384.

_ and — 1999: Time-dependent nonlinear Hadley circulation. J. Atmos. Sci., 56, 1797-1807.

Gill, A. E., 1980: Some simple solutions for heat-induced tropical circulation. Quart. J. Roy. Meteor. Soc., 106, 447-462.

Hack, J. J., W. H. Schubert, D. E. Stevens, and H.-C. Kuo, 1989 Response of the Hadley circulation to convective forcing in the ITCZ. J. Atmos. Sci., 46, 2957-2973.

Held, I. M., and A. Y. Hou, 1980: Nonlinear axially symmetric circulations in a nearly inviscid atmosphere. J. Atmos. Sci., 37, 515-533.

, and P. J. Phillips, 1990: A barotropic model of the interaction between the Hadley cell and a Rossby wave. J. Atmos. Sci., 47, $856-869$.

Hsu, C. J., and R. A. Plumb, 2000: Non-axisymmetric thermally driven circulations and upper tropospheric monsoon dynamics. J. Atmos. Sci., 57, 1254-1276.

Lindzen, R. S., and A. Y. Hou, 1988: Hadley circulation for zonally averaged heating centered off the equator. J. Atmos. Sci., 45, 2416-2427.
Neelin, J. D., 1988: Simple model for surface stress and low-level flow in the tropical atmosphere driven by prescribed heating. Quart. J. Roy. Meteor. Soc., 114, 747-770.

Plumb, R. A., and A. Y. Hou, 1992: The response of a zonally symmetric atmosphere to subtropical thermal forcing: Threshold behavior. J. Atmos. Sci., 49, 1790-1799.

Satoh, M., 1994: Hadley circulations in radiative-convective equilibrium in an axially symmetric atmosphere. J. Atmos. Sci., 51, 1947-1968.

Schneider, E. K., 1977: Axially symmetric steady-state models of the basic state for instability and climate studies. Part II. Nonlinear calculations. J. Atmos. Sci., 34, 280-296.

, 1987: Simplified model of the modified Hadley circulation. $J$. Atmos. Sci., 44, 3311-3328.

-, and R. S. Lindzen, 1977: Axially symmetric steady-state models of the basic state for instability and climate studies. Part I. Linearized calculations. J. Atmos. Sci., 34, 263-279.

Sobel, A. H., and C. S. Bretherton, 2000: Modeling tropical precipitation in a single column. J. Climate, 13, 4378-4392.

, J. Nilsson, and L. M. Polvani, 2001: The weak temperature gradient approximation and balanced tropical moisture waves. J. Atmos. Sci., 58, 3650-3665. 